\title{
Aneurysm of left ventricle at non-atherosclerosis lesion of coronary arteries
}

\author{
Olena Gogayeva*, Gennadiy Knishov, Anatolii Rudenko, Liudmyla Dzakhoieva, Sergiy Rudenko, \\ Kostyantyn Rudenko \\ From World Society of Cardiothoracic Surgeons 25th Anniversary Congress, Edinburgh \\ Edinburgh, UK. 19-22 September 2015
}

\section{Background/Introduction}

Aneurysm of left ventricle (ALV) is formed in each 5th patient with acute myocardial infarction in the presence of complete occlusion of the coronary artery $(\mathrm{CA})$ by atherosclerotic plaque. But we noticed the formation of ALV in the absence of atherosclerotic stenosis, for patients with myocardial "bridges" (MB). The essence of this anomaly is the presence of systolic compression of the tunneled segment of the artery, which in itself raises doubts about its clinical significance. Due to the attitude of the medical community toward the $\mathrm{MB}$, as a result of its ambiguous nature, and given the favorable long term trend, the MB is regarded as a variant of the norm. At the same time, increasing reports of cases of sudden death and myocardial infarction associated with the presence of MB demonstrates the relevance of this anomaly.

\section{Aims/Objectives}

To show possibility of formation of postinfarction aneurism of left ventricle (LV) in the absence of atherosclerotic plaques in CA.

\section{Method}

12 patients in average age $35+/-5$ years with transmural $\mathrm{MI}$ in anamnesis underwent standard examination (ECG, $\mathrm{ECHO}$ and angiography) and surgical treatment.

\section{Results}

All patients had ECG-signs of aneurysm of antero-septal and apical area of LV, which was confirmed by ECHO study, where we notice reduction of ejection fraction less $45 \%$ (from 35 till 45\%). On the coronary angiography we found myocardial "bridge" (MB) over middle portion of

Department of Surgical Treatment of Ischemic Heart Disease, GF "National Amosov's Institute of Cardiovascular Surgery NAMS of Ukraine", Kyiv, 03110, Ukraine 